DESY 01-185

\title{
PHYSICS AT HERA II
}

\author{
W. BUCHMÜLLER \\ Deutsches Elektronen-Synchrotron DESY, 22603 Hamburg, Germany \\ E-mail: buchmuwi@mail.desy.de
}

\begin{abstract}
Deep inelastic electron proton scattering at HERA II will allow precise studies of QCD and stringent tests of physics beyond the standard model. We discuss these two aspects of DIS with emphasis on the regime of high gluon densities at small $x$ and on scalar quark production in supersymmetric theories with broken R-parity.
\end{abstract}

\section{Introduction}

In deep inelastic scattering at HERA one studies the interactions of electrons with quarks in a wide range of momentum transfers $Q^{2}$ and center-of-mass energies $\sqrt{\hat{s}}=\sqrt{x s}$, up to $\sqrt{\hat{s}}_{\text {max }}=318 \mathrm{GeV}$. The 'Virtues of HERA' have been identified long agol. DIS at small $Q^{2}$ probes the structure of the proton and allows a variety of QCD tests. DIS at large $Q^{2}$ is sensitive to electroweak interactions, to a possible structure of quarks and electrons and, last but not least, to new particles and interactions predicted by extensions of the standard model.

HERA I has made important contributions to all of these topics. The increase of luminosity by a factor of 10 and the availability of longitudinal polarization for electrons and positrons at HERA II will widen the physics scope substantially. In the following we shall illustrate this with some examples concerning strong interactions, electroweak interactions and physics beyong the standard model. This complements the reports of the $\mathrm{H}^{2} \mathrm{~A}$ and ZEUSB collaborations on their physics program at HERA II. Detailed discussions can be found in the proceedings of previous HERA workshopst as well as in the review articles 6 .

\section{Beyond the Standard Model}

In connection with the Higgs mechanism of mass generation for vector bosons and fermions new physics beyond the standard model is expected at energies $\mathcal{O}(1 \mathrm{TeV})$. There are two classes of extensions of the standard model. In the first class a revolutionary change is predicted, either as quark-lepton com- 

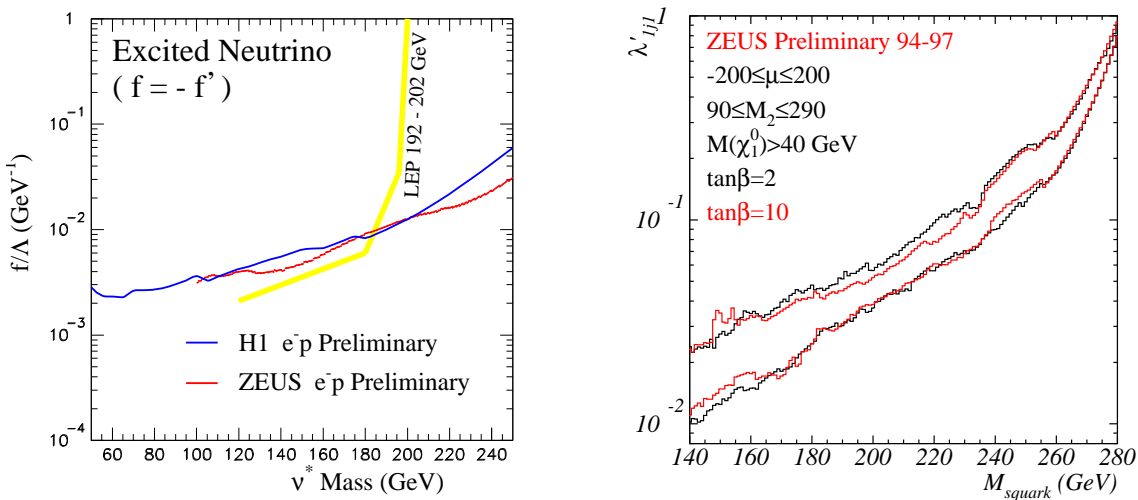

Figure 1. Bounds on coupling versus mass for excited neutrinos (left), and scalar quarks (right).

positeness or as manifestations of large extra dimensions. One then expects towers of excitations of the known elementary particles, either excited quarks and leptons or Kaluza-Klein excitations related to the TeV string scale. In the second class of extensions only a 'mild' modification of the standard model is predicted, the occurence of supersymmetry, where each particle aquires a superpartner. These extensions successfully predict the unification of all interactions at the GUT scale $\mathcal{O}\left(10^{16} \mathrm{GeV}\right)$. Via the seesaw mechanism they can also naturally account for the small neutrino masses indicated by the solar and atmospheric neutrino deficits.

HERA is particularly sensitive to those new particles for which single production is possible in ep-collisions. These include excited quarks and leptons, leptoquarks and scalar quarks in supersymmetric models with broken R-parity. For excited neutrinos the LEP limit of $200 \mathrm{GeV}$ could be significantly extended as shown on the left in fig. (11). On the right the range of upper limits on couplings of scalar quarks is shown as function of the squark mass for a class of supersymmetric models. The Yukawa couplings determine the production cross section, e.g. $\sigma\left(e_{R}^{+} d_{R} \rightarrow \widetilde{u}_{i}\right) \propto \lambda^{\prime 2}{ }_{1 i 1}$, with $\widetilde{u}_{1}=\widetilde{u}, \widetilde{u}_{2}=\widetilde{c}$, $\widetilde{u}_{3}=\widetilde{t}$.

Heavier particles beyond the kinematic reach of HERA lead to effective four-fermion interactions 9 with strength $4 \pi / \Lambda^{2}$. Depending on the chirality structure lower bounds on $\Lambda$ up to $9 \mathrm{TeV}$ have been obtained. For the vectorcurrent coupling the bound on $\Lambda$ can be interpreted as an upper bound on 

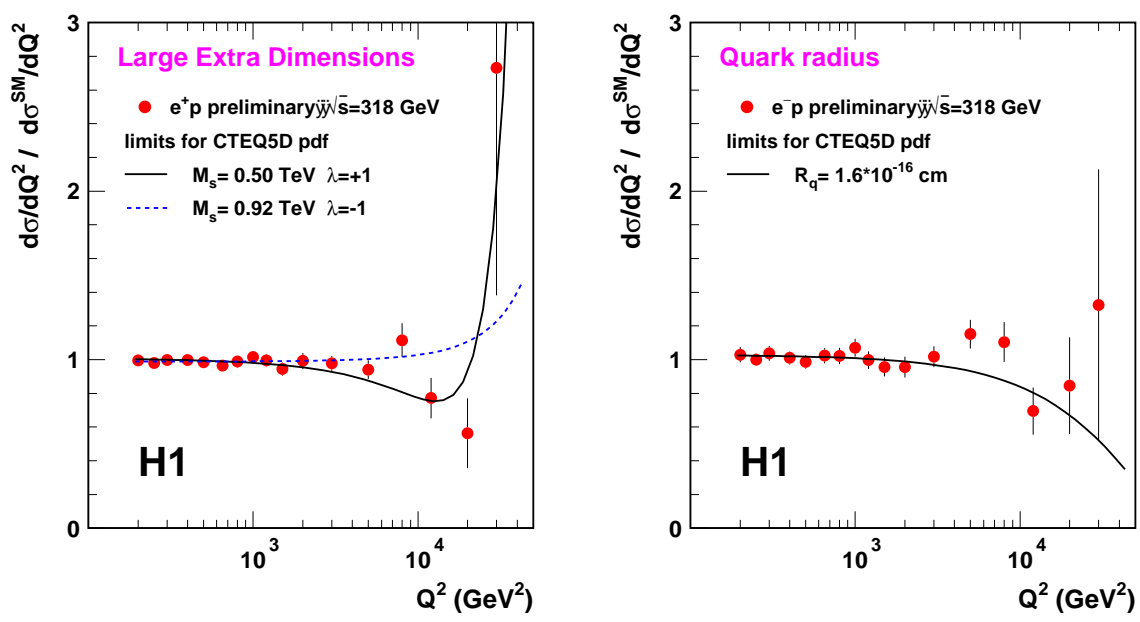

Figure 2. Effect of large extra dimensions of size $\sim 1 / M_{s}$ (left fnd $^{\text {and }}$ of a finite quark radius $R_{q}$ (right) on the neutral current DIS cross section. From ref. 10 .

the electromagnetic quark radius. The present limit is $R_{q}=1.6 \times 10^{-16} \mathrm{~cm}$ (cf. fig. (2)). Contact interactions are also induced by the Kaluza-Klein tower of gravitons in theories with large extra dimensions. The present bound on the corresponding mass scale is about $1 \mathrm{TeV}$ (cf. fig. (2)). At HERA II all these bounds can be improved by about a factor of three.

Supersymmetric theories with broken R-parity are for HERA the most promising extensions of the standard model11. In a class of these models Majorana neutrino masses are generated radiatively. It is then possible to relate the neutrino mixing matrix to squark production cross sections in epcollisions 12 . The analyses of the solar and atmospheric neutrino anomalies favour large neutrino mixings and therefore a neutrino mass matrix of the form

$$
m_{\nu i j}=m f_{i j}
$$

where $m<1 \mathrm{eV}$ and $f_{i j}=\mathcal{O}(1)$ for all $i, j=1 \ldots 3$. As we shall see, the large mixings among the neutrinos can lead to large couplings of electrons to new particles. The small value of the neutrino mass scale $m$ can be generated radiatively or by mass mixing via the seesaw mechanism.

In the supersymmetric standard model Yukawa interactions are described by the superpotential

$$
W=h_{e i j} E_{i}^{c} L_{j} H_{1}+h_{d i j} Q_{i} D_{j}^{c} H_{1}+h_{u i j} Q_{i} U_{j}^{c} H_{2},
$$


where $H_{1}$ and $H_{2}$ are two Higgs doublets with vacuum expectation values $v_{i}=\left\langle H_{i}\right\rangle, i=1,2$, and $v_{2} / v_{1}=\tan \beta$. The fact that the lepton doublets $L_{i}$ and the Higgs doublet $H_{1}$ have the same hypercharge, and therefore identical gauge quantum numbers, motivates the introduction of an additional R-parity violating part of the superpotential,

$$
W_{R}=\frac{1}{2} \lambda_{i j k} L_{i} L_{j} E_{k}^{c}+\lambda_{i j k}^{\prime} L_{i} Q_{j} D_{k}^{c} .
$$

The couplings $\lambda$ and $\lambda^{\prime}$ are in principle arbitrary. However, the same reason that leads to the introduction of these R-parity violating couplings also suggests the following connection between $\lambda, \lambda^{\prime}$ and the Yukawa couplings $h_{e}$ and $h_{d}$,

$$
\lambda_{i j k}=\lambda_{i} h_{e j k}^{T}+\lambda_{j} h_{e i k}^{T}, \quad \lambda_{i j k}^{\prime}=\lambda_{i}^{\prime} h_{d j k} .
$$

$W_{R}$ is then obtained from $W$ by a rotation among the fields $\left(L_{i}, H_{1}\right)$. Such a 'flavour alignment' suppresses the rates of flavour changing processes in the down quark sector $(\Delta S=1,2, \Delta B=1,2)$.

$W_{R}$ violates lepton number 13 . Hence, Majorana neutrino masses are induced,

$$
m_{\nu i j}=\lambda_{i}^{\prime} \lambda_{j}^{\prime} m_{\nu}^{(d)}+\lambda_{i} \lambda_{j} m_{\nu}^{(e)},
$$

where $m_{\nu}^{(d, e)}$ depends on the Yukawa couplings $h_{d, e}$ and the soft supersymmetry breaking parameters. In order to obtain the neutrino mass matrix (2) one needs $\lambda_{i}, \lambda_{j}^{\prime}=\mathcal{O}(1)$. The choice $\lambda_{i}=\lambda_{j}^{\prime}=1$ leads to the 'democratic' mixing matrix.

Predictions of this model with R-parity breaking are certain flavour changing processes, e.g. $B R\left(D^{0} \rightarrow \mu \mu, \mu e\right) \sim 10^{-5}$ and, in particular, the couplings for squark production, for instance,

$$
\lambda_{111}^{\prime} \sim \frac{m_{d}}{v} \tan \beta \sim 0.003, \quad \lambda_{122}^{\prime} \sim \frac{m_{s}}{v} \tan \beta \sim 0.05 .
$$

The value of $\lambda_{111}^{\prime}$ is consistent with the upper bound from neutrinoless double beta-decay. The present upper bound $44 \lambda_{122}^{\prime}<0.29$ based on $37 \mathrm{pb}^{-1}$ suggests that the sensitity of $\lambda_{122}^{\prime} \sim 0.05$ will be reached at HERA II.

\section{Electroweak Interactions}

DIS at large $Q^{2} \sim 10^{4} \mathrm{GeV}^{2}$ has so far tested electroweak unification, i.e., the approximate equality of neutral current and charged current cross section, 


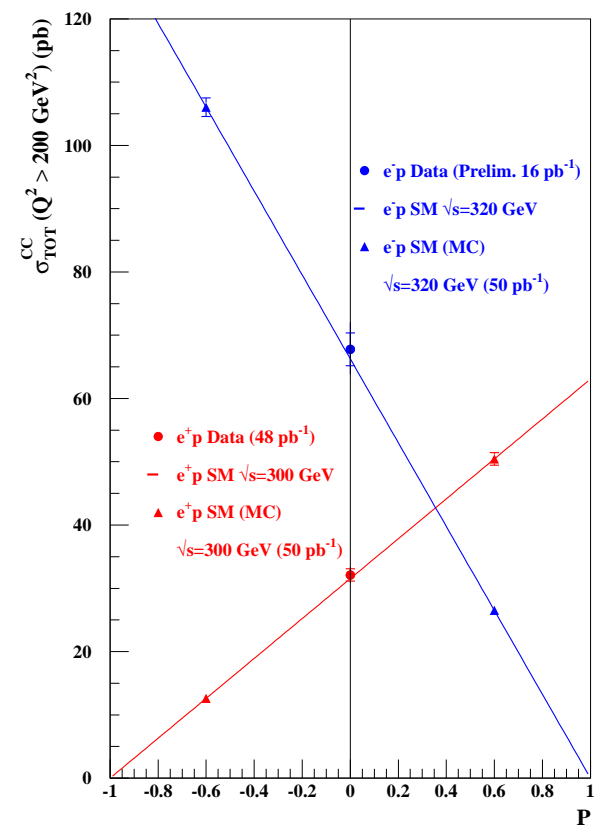

Figure 3. Charged current cross section as function of polarizatiqm $P$. Preliminary ZEUS data for $P=0$ and Monte Carlo simulations for $P \neq 0$. From ref. 15 .

$\sigma(N C) \sim \sigma(C C)$. At HERA II polarization will allow to test the classic prediction of the electroweak theory,

$$
\sigma^{C C}\left(e_{R}^{-} p\right)=\sigma^{C C}\left(e_{L}^{+} p\right)=0 .
$$

The exchange of right-handed $W_{R}$-bosons leads to a non-zero cross section. One expects a sensitivity to masses $m_{W_{R}} \simeq 600 \ldots 800 \mathrm{GeV}$, which corresponds to the present bounds from direct production and electroweak precision tests 16 . The expected precision for the charged current cross section obtained after one year of running is shown in fig. (3) as function of the degree of polarisation $P$.

The weak mixing angle $\sin ^{2}\left(\theta_{W}\right)\left(M_{Z}\right)$ can be determined from a measurement of the polarization asymmetry

$$
A\left(e_{L}^{-}-e_{R}^{-}\right)=\frac{d \sigma\left(e_{L}^{-}\right)-d \sigma\left(e_{R}^{-}\right)}{d \sigma\left(e_{L}^{-}\right)+d \sigma\left(e_{R}^{-}\right)} .
$$

For an integrated luminosity of $500 \mathrm{pb}^{-1}$ one expects an error of about 1\% 1 月. This is less accurate, but complementary to the LEP and SLC measurements. 
Polarization is also crucial to determine the properties of discovered new particles. For instance, for the scalar quarks discussed in the previous section one has

$$
\sigma\left(e_{R}^{+} p \rightarrow \widetilde{c} X\right) \propto{\lambda^{\prime 2}}_{122}^{2}, \quad \sigma\left(e_{L}^{+} p \rightarrow \widetilde{c} X\right)=0 .
$$

Such a measurement would prove that the discovered new scalar colour-triplet particle is the superpartner of a left-handed quark.

\section{Strong Interactions}

In deep inelastic scattering at HERA I many features of QCD have already been studied in great detail. These include

- proton and photon structure functions,

- jets and event shapes,

- determination of $\alpha_{s}$,

- hadronic final states,

- instanton induced processes,

- production of charm and bottom,

- vector meson production,

- diffractive processes.

All these quantities and processes will be studied with higher precisign at HERA II, and in particular the search for instanton induced processest 8 will be significantly improved.

In the following I shall concentrate on those aspects which have been most intriguing at HERA I. These are

1. the rapid rise of parton densities at small 219 , and

2. the large fraction of diffractive events at small $x$ 20 .

The rise of the structure functions at small $x$ has been anticipated based on the QCD renormalization group equations for moments 21 and the corresponding DGLAP evolution equations 22 for parton densities in the GRV mode23. In addition, at small $x$ large logarithms $\sim \alpha_{s} \ln 1 / x$ become important and have to be resummed. This is achieved by means of the BFKL 


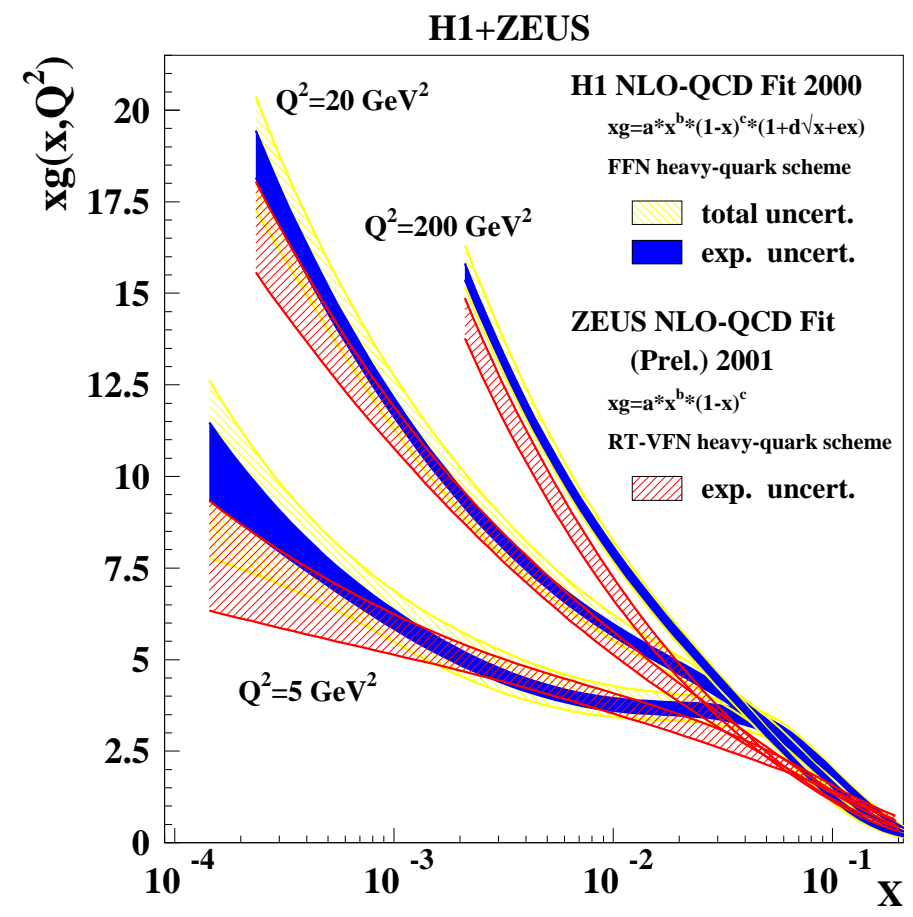

Figure 4. Gluon distribution function determined bo NLO-QCD fit to the structure function $F_{2}\left(x, Q^{2}\right)$ based on $\mathrm{H} 1$ data27 and ZEUS data28, respectively.

equation 24 which leads to the prediction of a power-like growth, $F_{2} \propto x^{-\lambda}$. The rapid rise at small $x$ reflects the strong radiation of gluons at high energies leading to large gluon densities. However, it still remains to be understood to what extent this rise can be described perturbatively and where it reflects non-perturbative effects, i.e., screening correction 25 and input parton distributions.

In order to identify possible non-perturbative effects, it is important to check how accurately the DGLAP approach describes different processes. A particularly interesting quantity is the gluon density which can be extracted from scaling violations of the structure functions,

$$
x g\left(x, Q^{2}\right) \propto \frac{\partial F_{2}\left(x, Q^{2}\right)}{\partial \ln Q^{2}} .
$$



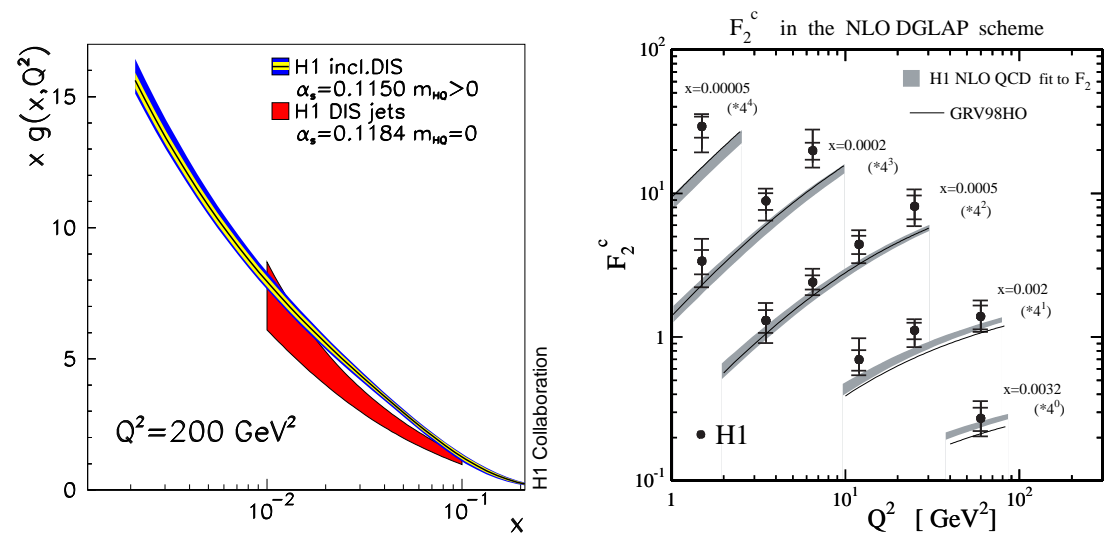

Figure 5. Gluon distribution function extracted from a NLO-QCD fit to dijet rates (left); the charm contribution to $F_{2}$ (right).

The gluon density extracted in a NLO analysis from the H1 and ZEUS data is shown in fig. (何) for different values of $Q^{2}$. The related determination of $\alpha_{s}$ has by now reached the remarkable precision 29 , 30 ,

$$
\begin{aligned}
& \alpha_{s}\left(M_{Z}^{2}\right)=0.1150 \pm 0.0017(\text { exp }) \pm 0.005(\text { theory })(H 1) \\
& \alpha_{s}\left(M_{Z}^{2}\right)=0.117 \pm 0.001(\text { stat }+ \text { uncorr }) \pm 0.005(\text { corr })(Z E U S, \text { prel. }) .
\end{aligned}
$$

Note, that the total error of the $\mathrm{H} 1$ result is dominated by the theoretical uncertainty.

Contrary to structure functions, the gluon density enters at leading order in the dijet cross section and in $F_{2}^{c}$, the charm contribution to the structure function $F_{2}$. In fig. (5) the NLO gluon density extracted from dijets is compared with the one determined from scaling violations; further, the corresponding prediction for $F_{2}^{c}$ is compared with data. It is clear that the higher accuracy at HERA II will lead to a stringent test of the DGLAP framework, which will require NNLO theoretical calculations for structure functions and jet cross sections.

A puzzling phenomenon in DIS at small $x$ is the occurence of a large fraction $\mathcal{O}(10 \%)$ of diffractive events 20 . This came as a big surprise for most experts in QCD, although it had been anticipated based on Regge theory 31 . However, the large rapidity gap events in DIS are difficult to understand in the parton picture to which almost everybody became used during the past 25 years because of the successes of perturbative QCD. 


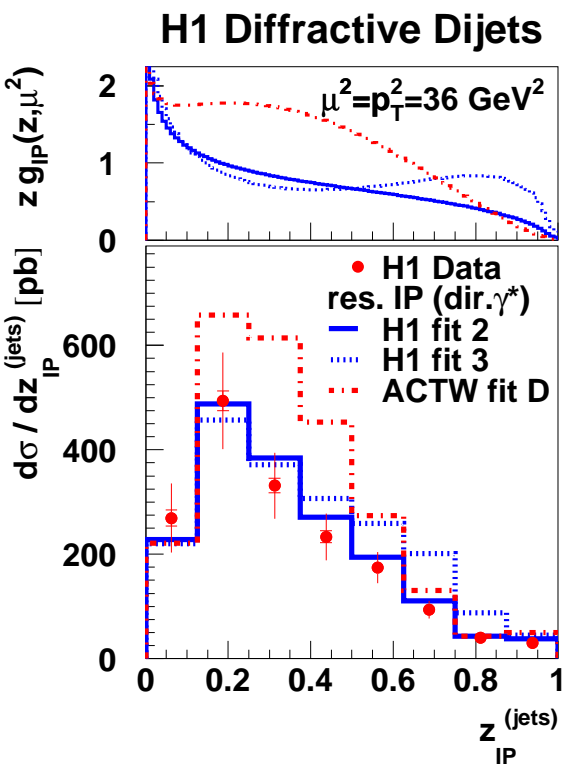

Figure 6. Diffractive dijet cross section as function of $z_{P}^{(j e t s)}(=\beta)$, compared with predictions based on different diffractive gluon densitips. The corresponding gluon densities $g\left(z, \mu^{2}\right)$ are shown in the upper pannel. From ref 37 .

We now have learned that, like inclusive structure functions, also diffractive structure functions can be expressed as convolution of parton cross sections with diffractive parton densities 32 . 33 . This factorization has been proven for inclusive and diffractive DIS with corresponding rigour 34 . The variation of the leading twist structure function with $Q^{2}$ is given by

$$
\begin{aligned}
& Q^{2} \frac{\partial}{\partial Q^{2}} F_{2}^{D}\left(\xi, \beta, Q^{2}\right)= \\
& 2 \sum_{q} e_{q}^{2} x \frac{\alpha_{s}}{2 \pi} \int_{\beta}^{1} \frac{d b}{b}\left(P_{q q}\left(\frac{\beta}{b}\right) \frac{d q\left(b, \xi, \mu^{2}\right)}{d \xi}+P_{q g}\left(\frac{\beta}{b}\right) \frac{d g\left(b, \xi, \mu^{2}\right)}{d \xi}\right) .
\end{aligned}
$$

Here $\xi \equiv x_{P}$ is the fraction of momentum lost by the proton, and $\beta=$ $Q^{2} /\left(Q^{2}+M^{2}\right)$ where $M$ is the diffractive mass, $M^{2}=(q+\xi P)^{2}$. For comparison, in inclusice DIS, $x=Q^{2} /\left(Q^{2}+W^{2}\right)$ where $W^{2}=(q+P)^{2}$ is the total invariant mass squared of the complete hadronic system. $d q / d \xi$ and $d g / d \xi$ are the diffractive quark and gluon densities, respectively, and $P_{q q}$ and $P_{q g}$ are the usual splitting functions. Note, that the $Q^{2}$-evolution affects only the 
H1 DATA (1994)

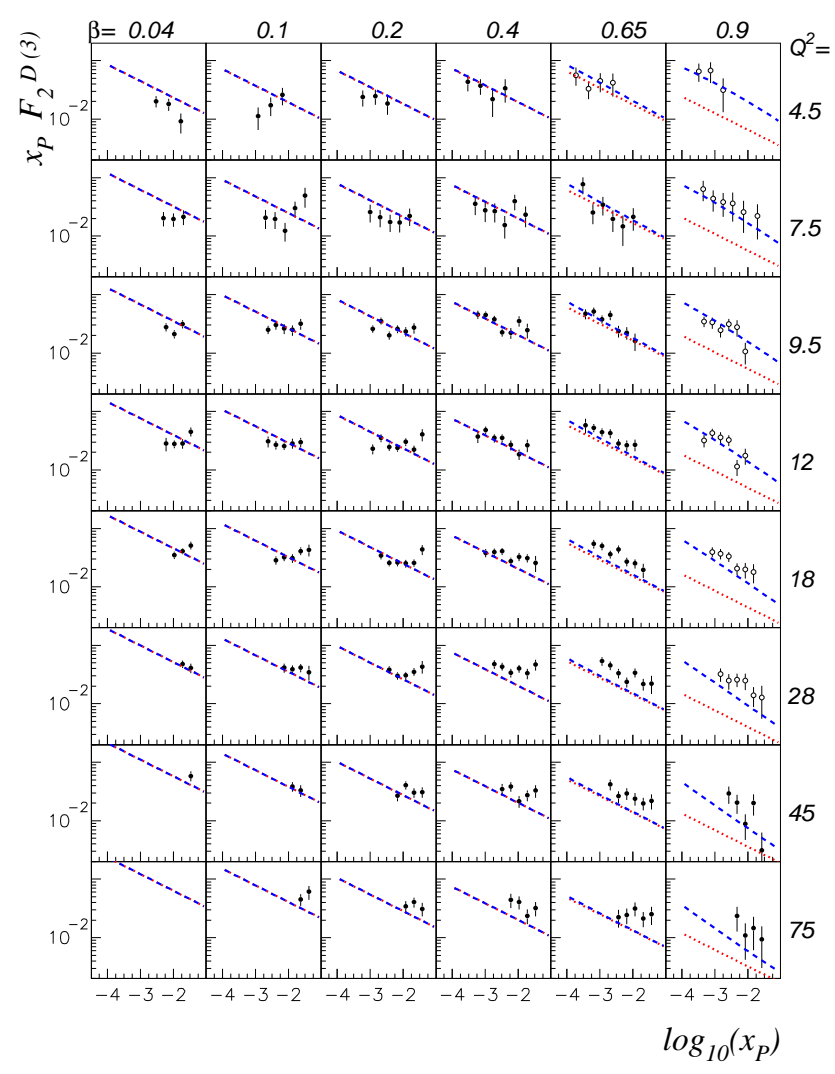

Figure 7. Prediction for the diffractive structure function $x_{P} F_{2}^{D(3)}$ compared with H1 data. The dashed lines correspond to the leading twist contribution with the twistadded. The leading twist contribution is shown by the dotted lines. From ref. 40 .

$\beta$-dependence of the structure function and not the $\xi$-dependence. Hence, the $\xi$-dependence is an entirely non-perturbative property of the proton. It corresponds to the dependence on the total hadronic energy $W$ for fixed $\beta$, since $W^{2} \simeq Q^{2} / x=Q^{2} /(\xi \beta)$. In models with Regge factorization 35 the $\xi$ dependence is given by Regge theory. For the diffractive gluon density one has $d g\left(\xi, \beta, Q^{2}\right) / d \xi \propto \xi^{1-2 \alpha_{P}(0)} g\left(\beta, Q^{2}\right)$. 


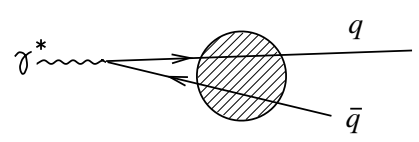

a)

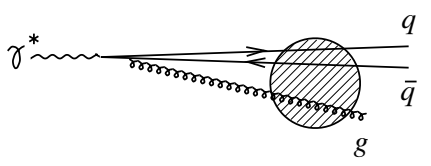

b)
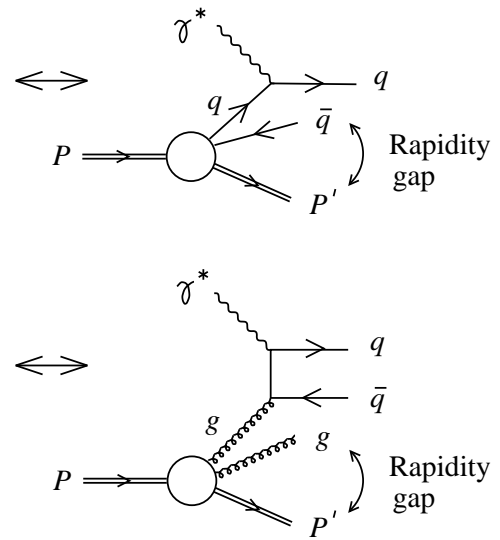

Figure 8. Diffractive DIS in the proton rest frame (left) and the Breit frame (right); asymmetric quark fluctuations correspond to diffractive quark scattering, asymmetric gluon fluctuations to diffractive boson-gluon fusion.

Diffractive DIS can be analyzed in analogy to inclusive DIS36. The diffractive structure function yields the diffractive quark distribution. Its scaling violation, and also diffractive dijet and charm production determine the diffractive gluon density. In fig. (6) the measured dijets rates (lower panel) are compared with predictions based on different diffractive gluon densities $g\left(z, \mu^{2}\right)$ (upper panel) which have been extracted from fits to the diffractive structure function. At present quantitative tests are just beginning. From HERA II we can expect a precise determination of the diffractive gluon depsity. Here also higher twist effects have to be taken into account at large $\beta 3330$. The results of a recent analysis including such effects are shown in fig. (7).

What do we learn from a determination of diffractive quark and gluon densities? A comparison of inclusive and diffractive DIS is particularly interesting in the proton rest frame 41 . Here diffractive and non-diffractive processes can be understood as scattering of partonic fluctuations of the photon on the proton. The diffractive quark and gluon densities then correspond to asymmetric quark and gluon fluctuations (cf. fig. (8) projected onto the colour singlet state. The formation of the final state is a non-perturbative phenomenon which depends on soft momenta and the properties of confinement. Hence, a comparison of diffractive and non-diffractive processes should help to understand non-perturbative properties of the proton. Correspondingly, one expects that at small $x$ diffractive and non-diffractive processes have a 

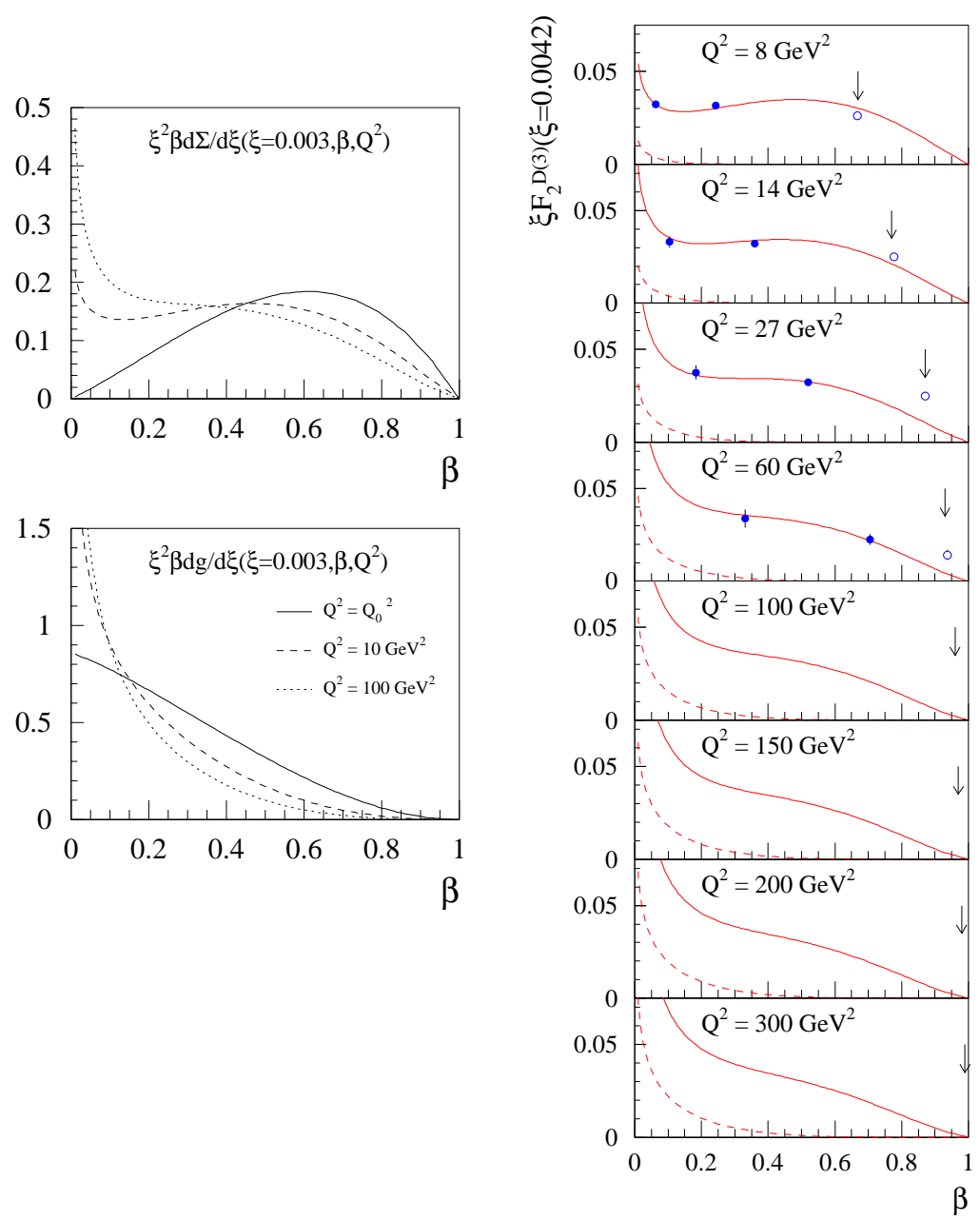

Figure 9. Diffractive singlet quark and gluon distributions at an initial scale $Q_{0}^{2}$ and after $Q^{2}$ evolution (left) diffractive structure function $F_{2}^{D(3)}$ for different values of $Q^{2}$ compared with ZEUS data 49. The arrows indicate the ralue of $\beta$ beyond which higher twist terms are expected to become important. From ref. 4 .

similar dependence on the total hadronic energy $W 42$.

An interesting toy model for a comparison of inclusive and diffractive DIS is a large hadronic target 4344 . In this model the cross section for a dipole of 
transverse size $y$ is of the Glauber-type 45

$$
\sigma(y)=\sigma_{0}\left(1-e^{-a y^{2}}\right) .
$$

The corresponding diffractive 46 and inclusive 40,47 parton densities have been explicitly calculated. The model can also be used to estimate saturation and higher twist effect.48.

For the diffractive quark and gluon densities one obtains 46

$$
\begin{gathered}
\xi^{2} \frac{d q\left(\beta, \xi, Q_{0}^{2}\right)}{d \xi}=\frac{a \Omega N_{c}(1-\beta)}{2 \pi^{3}} f_{q}(\beta), \\
\xi^{2} \frac{d g\left(\beta, \xi, Q_{0}^{2}\right)}{d \xi}=\frac{a \Omega N_{c}^{2}(1-\beta)^{2}}{2 \pi^{3} \beta} f_{g}(\beta) .
\end{gathered}
$$

Here $\Omega$ is the transverse size of the target, $N_{c}$ is the number of colours, and $Q_{0}$ is the scale where the model calculation is matched to the perturbative evolution. A non-trivial dependence of the parton densities on $\xi=x_{P}$ may be introduced by assuming a $\xi$-dependence either of the transverse size 46 , i.e., $\Omega=\Omega(\xi)$, or of the saturation scale $4 a=a(\xi)$, leading to the same phenomenology. Note, that the functions $f_{q}(\beta)$ and $f_{g}(\beta)$ are parameter free predictions of the model. The singlet quark density $d \Sigma / d \xi=6 d q / d \xi$ and the gluon density $d g / d \xi$ are shown in fig. (9). Their $\beta$-dependence is an interesting non-perturbative property of the proton.

Fig. (9) also shows a comparison between a theoretical prediction 46 for the diffractive structure function and the presently available ZEUS data 49 . Further data from HERA I and HERA II will allow an extension to larger values of $Q^{2}$ where theory predicts almost no change. It will be important to check whether the DGLAP approach is indeed correct for the diffractive structure function. Further, because of the high statistics at HERA II it will be possible to determine the diffractive gluon density also from dijet and charm production. One can then study the non-perturbative dependence on $\xi$ and the normalization relative to the inclusive gluon density. Particularly interesting will be the comparison of event shapes in inclusive and diffractive DIS. In this way we will get far the first time some insight into the regime of high gluon densities in QCD 50 .

\section{Summary}

The increase in luminosity at HERA II will allow precise measurements of various observables and processes in QCD. Particularly interesting is the determination of $\alpha_{s}$, where already now the precision of LEP has almost been 
reached. The comparison of the measured running coupling $\alpha_{s}\left(\mu^{2}\right)$ with lattice calculations will lead to an important quantitative test of QCD. Further, the search for instantons can be improved, and one can test the DGALP framework by comparing determinations of the gluon density from different processes. In this way, HERA can probe the regime of high gluon densities in a clean and controlable way.

The high luminosity and polarization will also lead to new tests of the electroweak theory. Searches for physics beyond the standard model will be significantly improved, in particular with respect to scalar quark production in models with R-parity breaking. Clearly, this sensitivity will also allow the discovery of phenomena not anticipated in this contribution.

\section{Acknowledgments}

I would like to thank T. Gehrmann, K. Golec-Biernat, D. Haidt, M. Klein, M. Kuze, P. Schleper and G. Wolf for their help in the preparation of this contribution and the organizers of DIS2001 for the splendid hospitality in Bologna.

\section{References}

1. L. Maiani, The Virtues of HERA, in Proc. Experimentation at HERA, Amsterdam 1983, DESY HERA 83/20

2. E. Elsen, these proceedings

3. B. Foster, these proceedings

4. Proc. The HERA Workshop, Vol. I,II, Hamburg 1987, ed. R. D. Peccei; Proc. Physics at HERA, Vol. I,II,III, Hamburg 1991, eds. W. Buchmüller, G. Ingelman;

Proc. Future Physics at HERA, Hamburg 1996, eds. G. Ingelman, A. De Roeck, R. Klanner

5. H. Abramowicz, A. Caldwell, Rev. Mod. Phys. 71 (1999) 1275

6. G. Wolf, hep-ex/0105055

7. H1 Collaboration, Paper 956 submitted to ICHEP 2000, Osaka, Japan ZEUS Collaboration, Paper 1040 submitted to ICHEP 2000, Osaka, Japan

8. ZEUS collaboration, abstract 1042, submitted to ICHEP00, Osaka

9. For a recent review and references, see

M. Kuze, Searches for new physics at HERA, hep-ex/0106030

10. H1 Collaboration, C. Adloff et al., Phys. Lett. B 479 (2000) 358;

H1 Collaboration, Abstract 951 submitted to ICHEP00, Osaka, Japan 
11. E. Perez, Y. Sirois, H. Dreiner, Proc. Future Physics at HERA, Hamburg 1996, eds. G. Ingelman, A. De Roeck, R. Klanner, p. 297

12. W. Buchmüller, R. Rückl, in preparation

13. For a recent discussion and references, see A. de Gouvêa, S. Lola, K. Tobe, Phys. Rev. D 63 (2001) 035004

14. H1 Collaboration, C. Adloff et al., Eur. Phys. J. C 20 (2001) 639

15. T.Fusayasu, Moriond Conf. on Electroweak Interactions, Les Arcs, 2001

16. Review of Particle Physics, Eur. Phys. J. C 15 (2000) 1

17. J. Blümlein, M. Klein, T. Riemann, in Proc. The HERA Workshop, Vol. II, Hamburg 1987, ed. R. D. Peccei, p.687;

D. Haidt, private communication

18. A. Ringwald, F. Schrempp, Phys. Lett. B 503 (2001) 331

19. H1 Collaboration, I. Abt et al., Nucl. Phys. B 407 (1993) 515; ZEUS Collaboration, M. Derrick et al., Phys. Lett. B 316 (1993) 412

20. ZEUS Collaboratin, M. Derrick et al., Phys. Lett. B 315 (1993) 481; H1 Collaboration, T. Ahmed et al., Nucl. Phys. B 429 (1994) 477

21. A. de Rujula et al., Phys. Rev. D 10 (1974) 1649

22. V. N. Gribov, L. N. Lipatov, Sov. J. Nucl. Phys. 15 (1972) 438, 675;

G. Altarelli, G. Parisi, Nucl. Phys. B 126 (1977) 298;

Yu. L. Dokshitzer, Sov. Phys. JETP 46 (1977) 641

23. M. Glück, E. Reya, A. Vogt, Phys. Lett. 306 B (1993) 391

24. L. N. Lipatov, Sov. J. Nucl. Phys. 23 (1976) 338;

V. S. Fadin, E. A. Kuraev, L. N. Lipatov, Phys. Lett. 60 B (1975) 50; Sov. Phys. JETP 44 (1976) 443; ibid. 45 (1977) 199;

Y. Y. Balitski, L. N. Lipatov, Sov. J. Nucl. Phys. 28 (1978) 822

25. L. V. Gribov, E. M. Levin, M. G. Ryskin, Phys. Rep. 100 (1993) 481; for a review and references, see

A. H. Mueller, Proc. QCD: Perturbative or nonperturbative?, Autumn School Lisbon 1999, hep-ph/9911289

26. For a recent discussion and references, see G. Altarelli, R. Ball, S. Forte, Nucl. Phys. B 599 (2001) 383

27. H1 Collaboration, R. Wallny, these proceedings

28. ZEUS Collaboration, K. Nagano, these proceedings

29. H1 Collaboration, C. Adloff et al., Eur. Phys. J. C 21 (2001) 33

30. ZEUS Collaboration, A. Cooper-Sarkar, these proceedings

31. A. Donnachie, P. V. Landshoff, Phys. Lett. B 191 (1987) 309

32. L. Trentadue, G. Veneziano, Phys. Lett. B 323 (1994) 201

33. A. Berera, D. E. Soper, Phys. Rev. D 50 (1994) 4328

34. J. C. Collins, Phys. Rev. D 57 (1998) 3051

35. G. Ingelman, P. E. Schlein, Phys. Lett. B 152 (1985) 256 
36. T. Gehrmann, W. J. Stirling, Z. Phys. C 70 (1996) 89

37. H1 Collaboration, C. Adloff et al., Eur. Phys. J. C 20 (2001) 29

38. J. Bartels, J. Ellis, H. Kowalski, M. Wüsthoff, Eur. Phys. J. C 7 (1999) 443

39. A. Hebecker, T. Teubner, Phys. Lett. B 498 (2001) 16

40. K. Golec-Biernat, M. Wüsthoff, Eur. Phys. J. C 20 (2001) 313

41. J. D. Bjorken, AIP Conf. Proc. No.6, eds. M. Bander et al. (AIP, New York, 1972) p. 151;

J. D. Bjorken, J. B. Kogut, Phys. Rev. D 8 (1973) 1341

42. W. Buchmüller, A. Hebecker, Nucl. Phys. B 476 (1996) 203;

W. Buchmüller, Phys. Lett. B 353 (1995) 335

43. L. McLerran, R. Venugopalan, Phys. Rev. D 49 (1994) 2233

44. A. Hebecker, H. Weigert, Phys. Lett. B 432 (1998) 215

45. N. N. Nikolaev, B. G. Zakharov, Z. Phys. C 49 (1991) 607

46. W. Buchmüller, T. Gehrmann, A. Hebecker, Nucl. Phys. B 537 (1999) 477

47. A. H. Mueller, Nucl. Phys. B 558 (1999) 285

48. K. Golec-Biernat, M. Wüsthoff, Phys. Rev. D 59 (1999) 014017; ibid. Phys. Rev. D 60 (1999) 114023

49. ZEUS Collaboration, J. Breitweg et al., Eur. Phys. J. C 6 (1999) 43

50. J. Jalilian Marian, A. Kovner, A. Leonidov, H. Weigert, Nucl. Phys. B 504 (1997) 415;

A. Kovner, J. G. Milhano, H. Weigert, Phys. Rev. D 62 (2000) 114005 

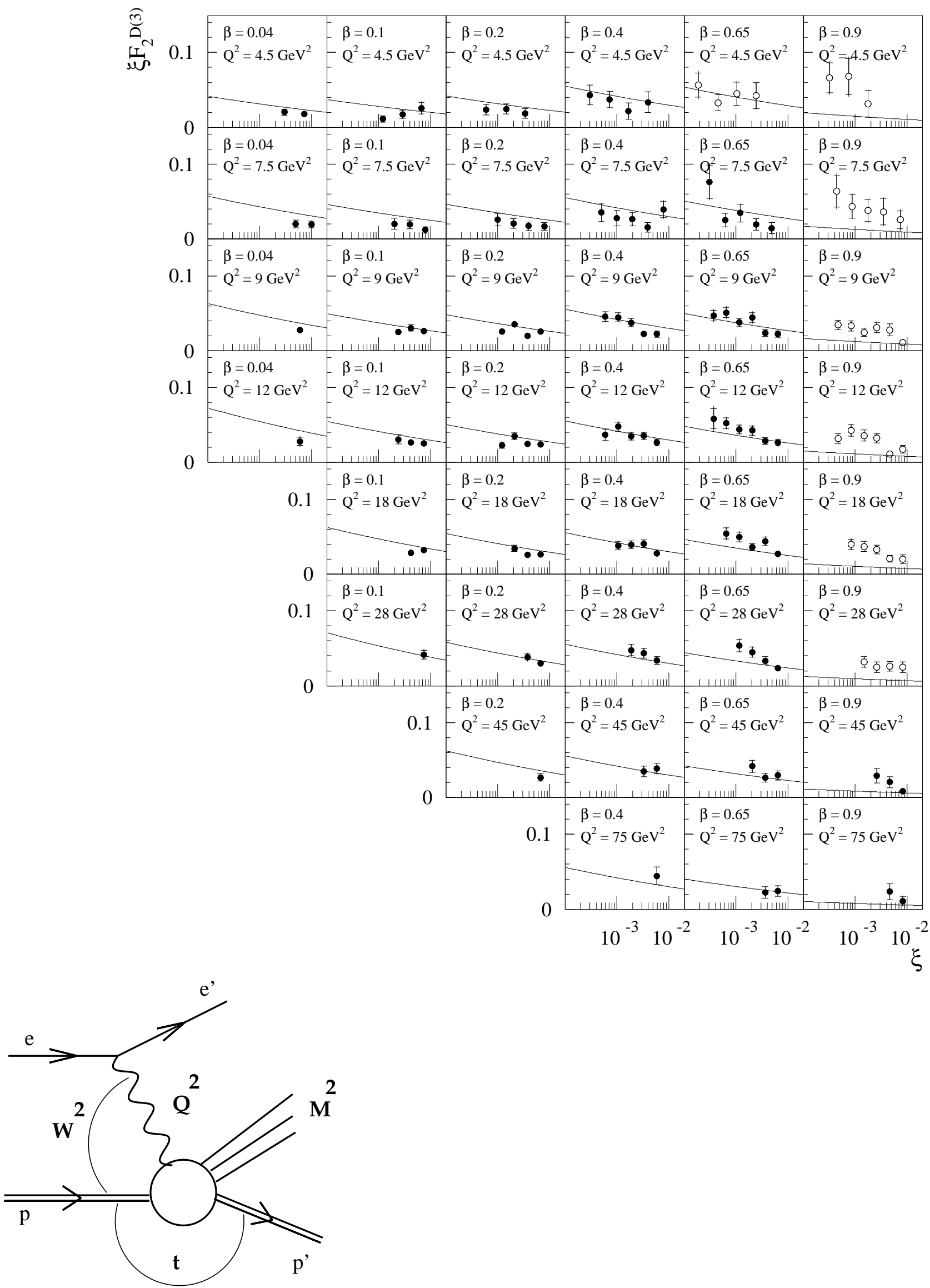


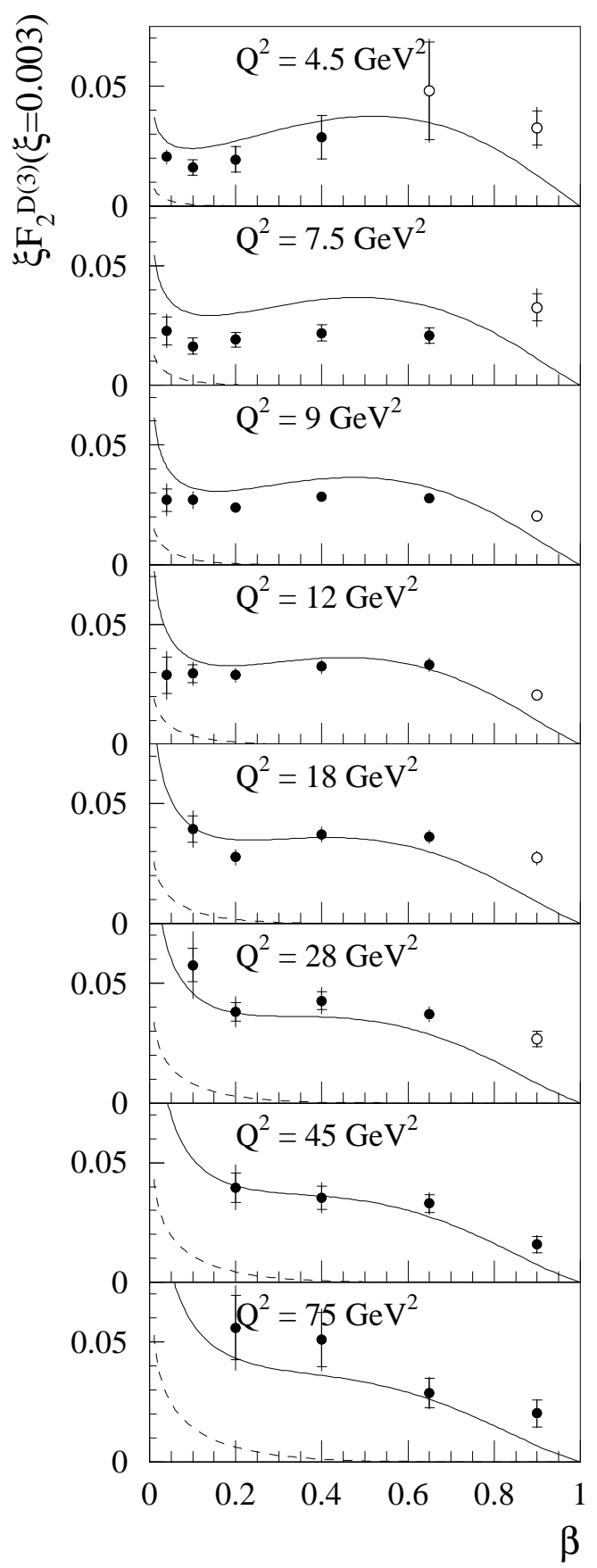




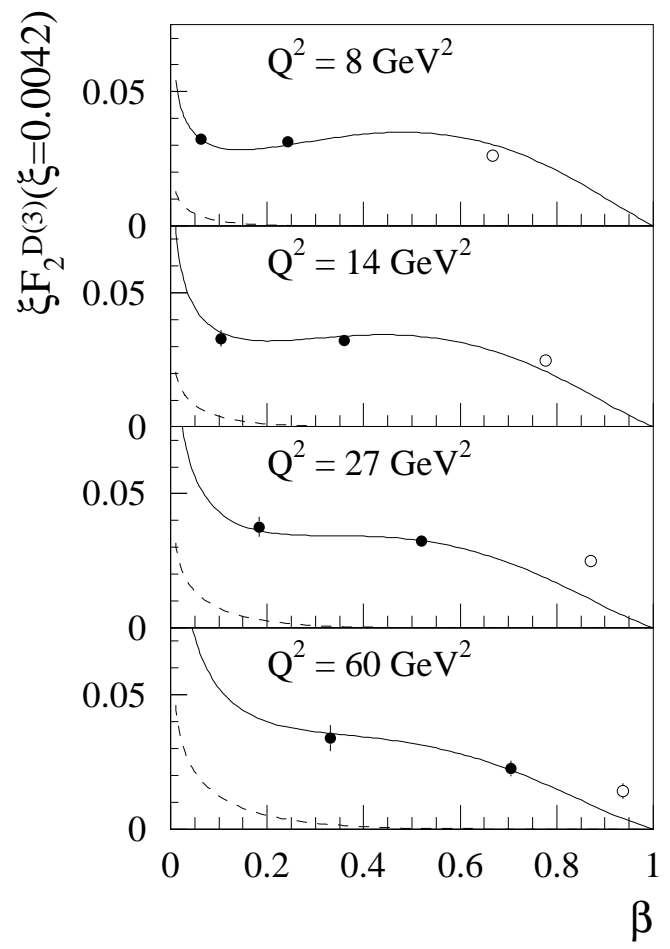




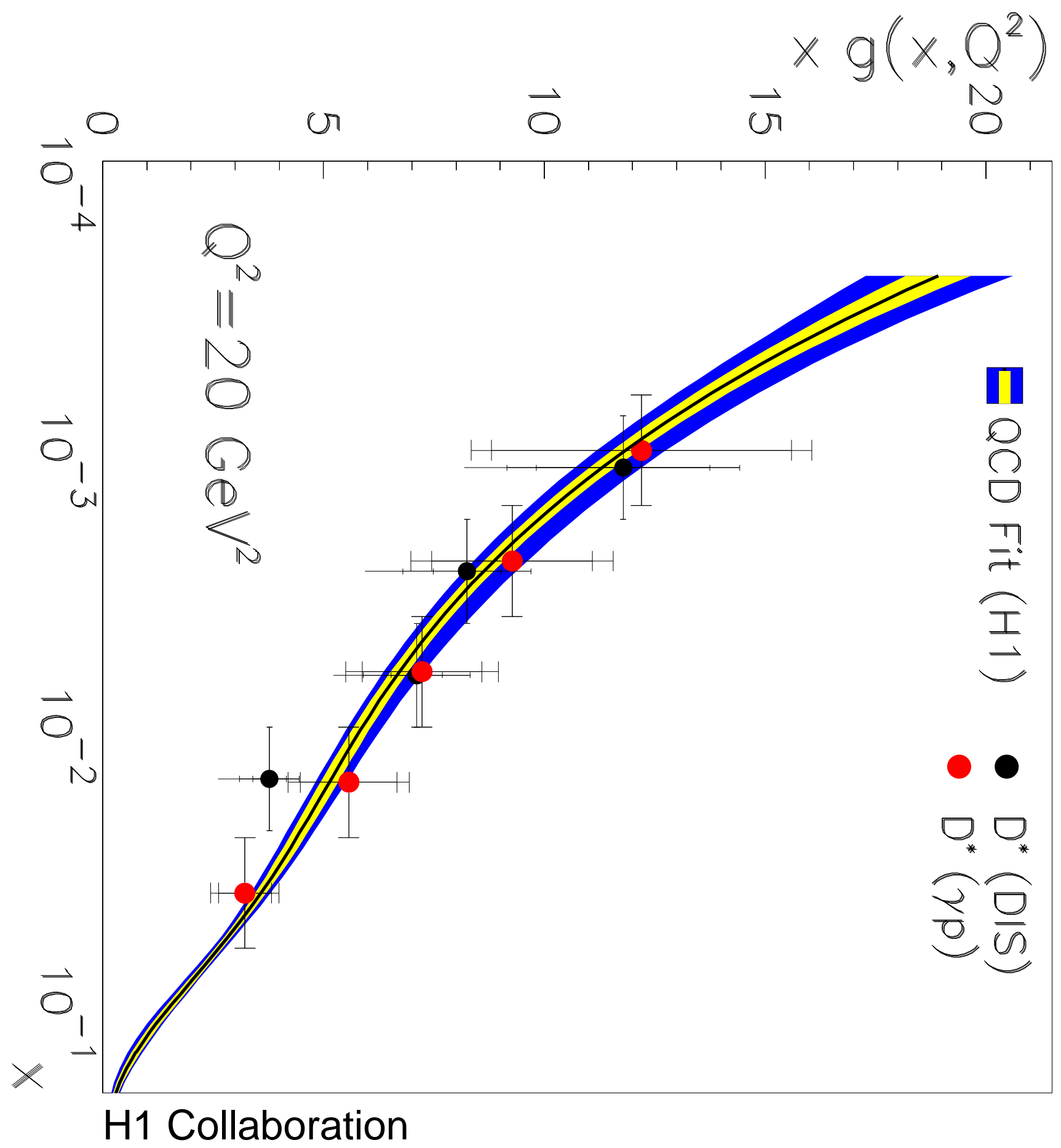




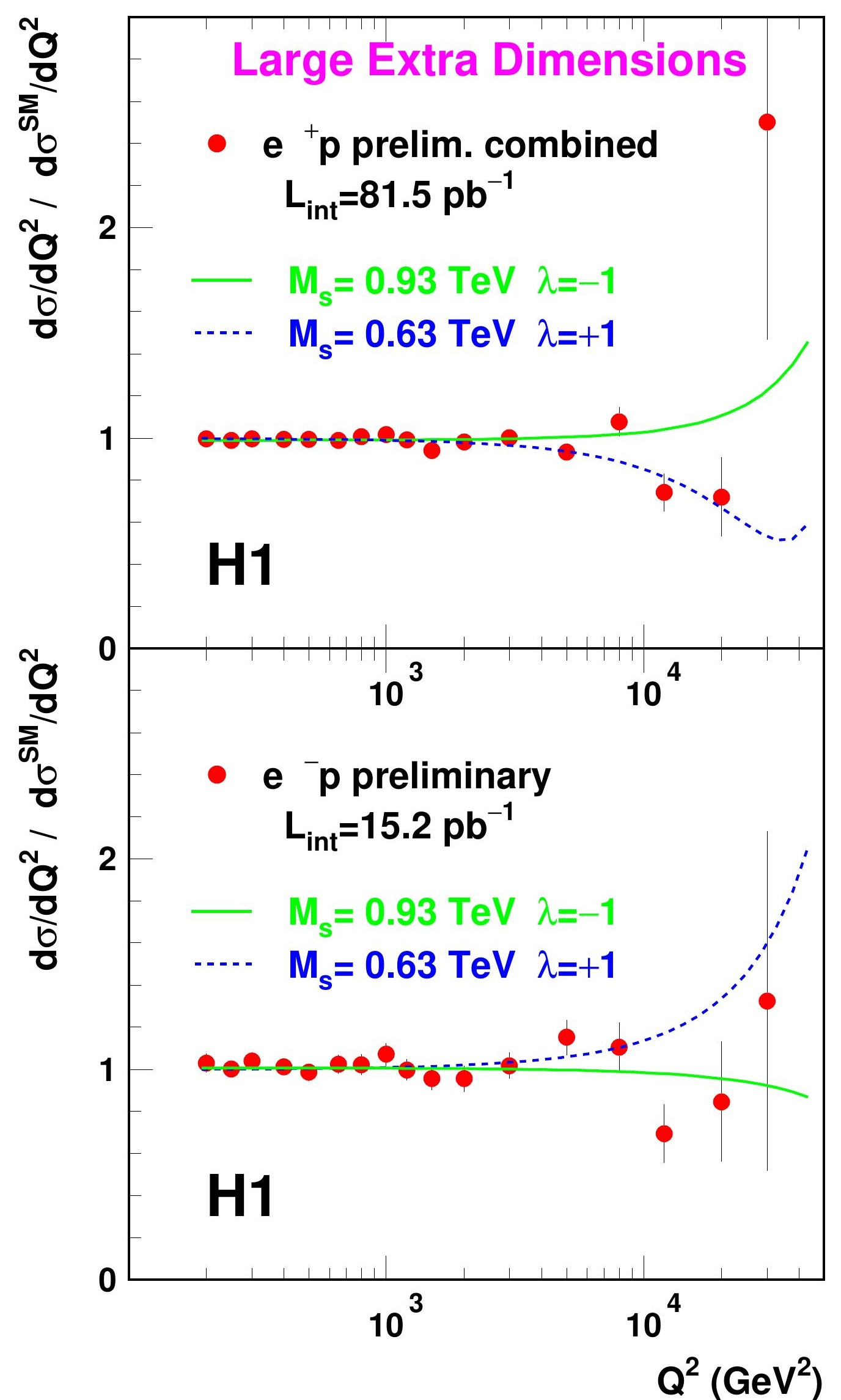




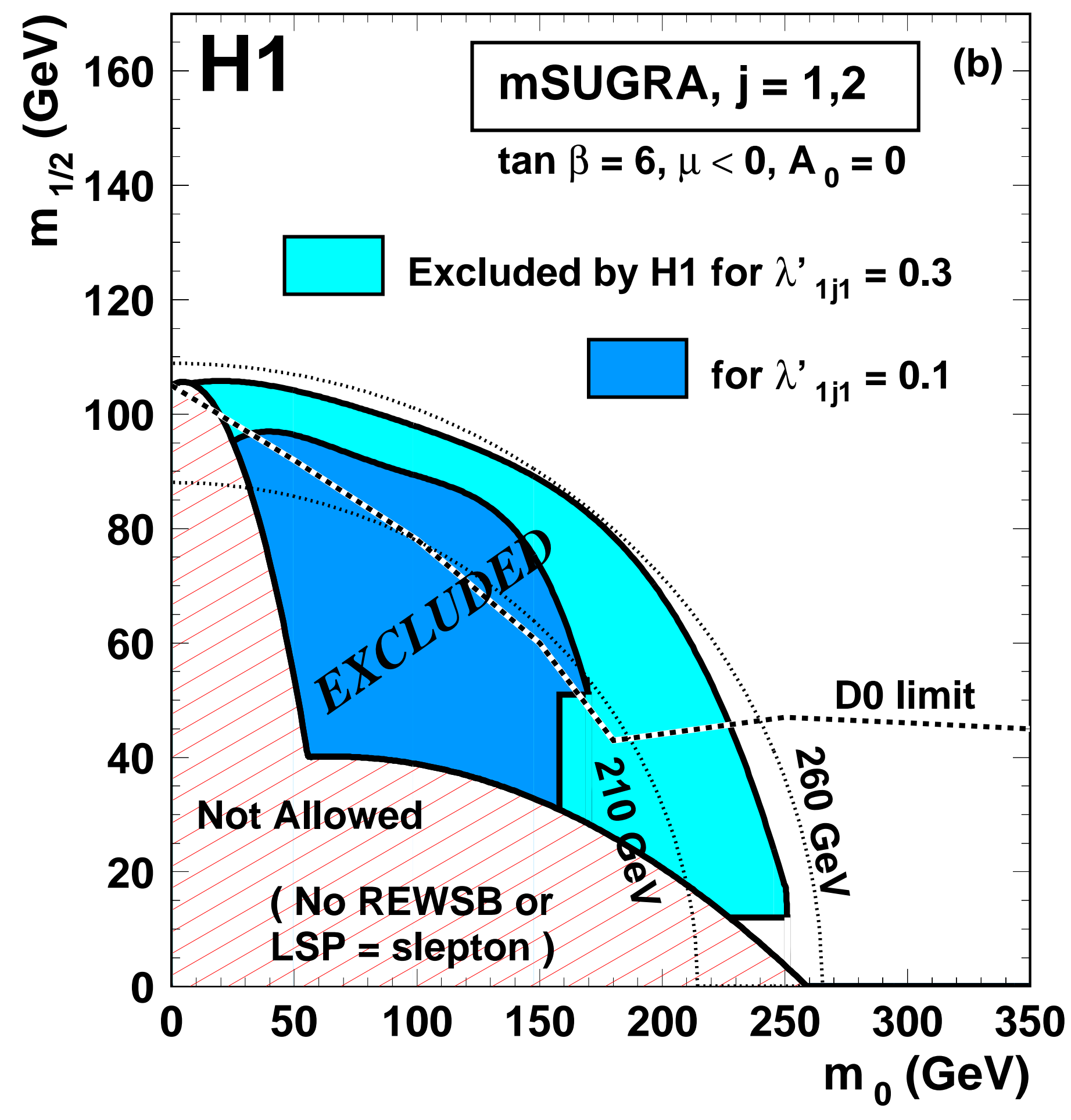

